\title{
Neural Combinatory Constituency Parsing
}

\author{
Zhousi Chen, Longtu Zhang, Aizhan Imankulova, and Mamoru Komachi \\ Tokyo Metropolitan University \\ 6-6 Asahigaoka, Hino, Tokyo 191-0065, Japan \\ chen-zhousided.tmu.ac.jp vincentzlteoutlook.com \\ aizhan.imankulova@cogsmart-global.com komachi@tmu.ac.jp
}

\begin{abstract}
We propose two fast neural combinatory models for constituency parsing: binary and multibranching. Our models decompose the bottomup parsing process into 1) classification of tags, labels, and binary orientations or chunks and 2) vector composition based on the computed orientations or chunks. These models have theoretical sub-quadratic complexity and empirical linear complexity. The binary model achieves an F1 score of 92.54 on Penn Treebank, speeding at 1327.2 sents/sec. Both the models with XLNet provide near state-of-theart accuracies for English. Syntactic branching tendency and headedness of a language are observed during the training and inference processes for Penn Treebank, Chinese Treebank, and Keyaki Treebank (Japanese).
\end{abstract}

\section{Introduction}

Transition-based and chart-based methods are two main paradigms for constituency parsing. Transition-based parsers (Dyer et al., 2016; Kitaev and Klein, 2020) build a tree with a sequence of local actions. Despite their $O(n)$ computational complexity, the locality makes them less accurate and necessitates additional grammars or lookahead features for improvement (Kuhlmann et al., 2011; Zhu et al., 2013; Liu and Zhang, 2017c). By contrast, chart-based parsers are conceptually simple and accurate when used with a CYK-style algorithm (Kitaev and Klein, 2018; Zhou and Zhao, 2019) for finding the global optima. However, their complexity is $O\left(n^{3}\right)$. To achieve both accuracy and simplicity (without high complexity) is a critical problem in parsing.

Recent efforts were made using neural models. In contrast to earlier symbolic approaches (Charniak, 2000; Klein and Manning, 2003), neural models are simplified by utilizing their adaptive distributed representation, thereby eliminating compli-

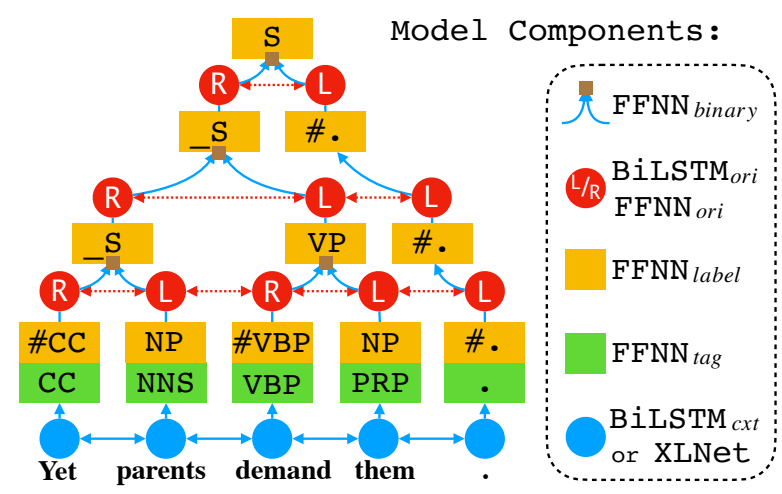

Figure 1: Parsing instance with the binary model. The bottom-up flow of word information is indicated by blue arrows and orientation flows by dotted red arrows. Binary parsing explores the internal constituents of S. Special labels prefixed with “\#” or "_," are sub category placeholders caused by binarization and stratification.

cated symbolic engineering. The seq2seq model for parsing (Vinyals et al., 2015) leverages such representation to interpret the structural task as a general sequential task. With augmented data and ensemble, it outperforms the symbolic models mentioned in Petrov et al. (2006) and provides a complexity of $O\left(n^{2}\right)$ with the attention mechanism (Bahdanau et al., 2015). However, its performance is inferior to those of specialized neural parsers (Liu and Zhang, 2017a,b,c). Socher et al. (2013) proposed a parsing strategy for a symbolic constituent parser augmented with neural vector compositionality. It did not outperform the two paradigms in neural style probably because the neural techniques, such as contextualization, are not fully exploited. Kitaev and Klein (2020) showed that a simple transitionbased model with a dynamic distributed representation, BERT (Devlin et al., 2019), nearly delivers a state-of-the-art performance.

We propose a pair of greedy combinatory parsers (i.e., neural combinators) that efficiently utilize vector compositionality with recurrent components to 


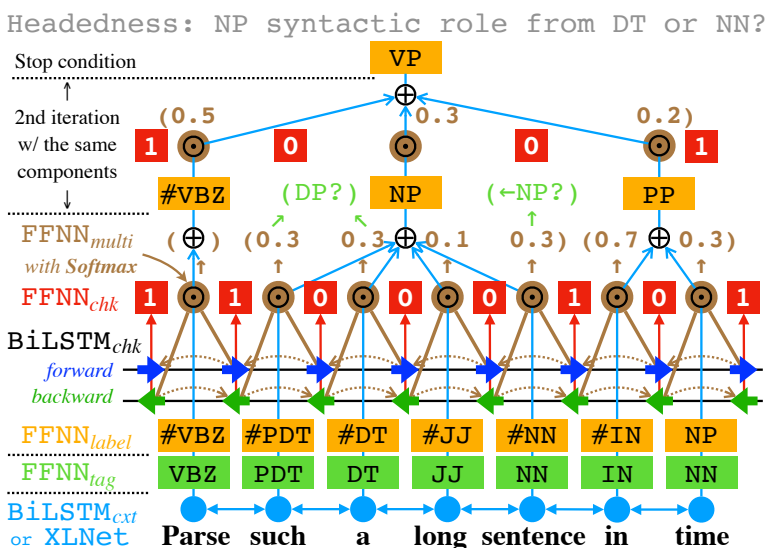

Figure 2: Multi-branching parsing uses chunks instead of orientations to form constituents. Chunks impose Softmax-normalized weights for their inputs. The unsupervised weights provide a shred of evidence for the headedness problem (Zwicky, 1985).

address the aforementioned issues. Their bottomup parsing process is a recursive layer-wise loop of classification and vector composition, as illustrated in Figures $1 \& 2$. Both parsers work on multiple unfolded variable-length layers, iteratively combining vectors until one vector remains. The binary model provides either left or right orientation for each word or constituent, whereas the multi-branching model marks chunks as constituents at their boundaries. Constituent embeddings are composed based on orientations or chunks. Tagging and labeling are directly performed on all composed embeddings, creating the elements for building a tree: tags, labels, and paths. The deterministic and greedy characteristics yield two simple and fast models, and they investigate different linguistic aspects.

The contributions of our study are as follows:

- We propose two combinatory parsers ${ }^{1}$ at $O(n)$ average-case complexity with a theoretical $O\left(n^{2}\right)$ upper bound. The binary parser achieves a competitive F1 score on Penn Treebank. Both models are the fastest and yet more compact than many previous models.

- We extend the proposed models with a recent pre-trained language model, XLNet (Yang et al., 2019). These models have higher speeds and are comparable to state-of-the-art parsers.

- The binary model leverages Chomsky normal form (CNF) factors as a training strategy

\footnotetext{
${ }^{1}$ Our code, visualization tool, and pre-trained models are available at https://github.com/tmu-nlp/nccp
}

and reflects the branching tendency of a language. The multi-branching model reveals constituent headedness (Zwicky, 1985) with an attention mechanism.

\section{Previous Work}

Transition-based parsers. A transducer takes sequential lexical inputs and produces sequential tree-constructing actions in $O(n)$ time. Although it can perfectly parse formal languages, complex semantics and long dependencies make it difficult to parse natural languages. Informative features (Liu and Zhang, 2017c; Kitaev and Klein, 2020; Yang and Deng, 2020), or training and decoding strategies such as dynamic oracles (Cross and Huang, 2016), reranking (Charniak and Johnson, 2005), beam search, and ensemble, can increase the accuracy. However, these make the models complex, and the paradigm fails to naturally parallelize actions.

Chart-based parsers. An exhaustive search algorithm checks every possibility in a triangular chart and finds the optimal tree globally. Recent neural chart parsers have achieved state-of-the-art accuracy (Kitaev and Klein, 2018; Zhou and Zhao, 2019; Mrini et al., 2020; Zhang et al., 2020). Despite their high accuracy, they are comparatively inefficient. Only $2 n-1$ of $O\left(n^{2}\right)$ scoring nodes in the chart contain true constituents; many are filler nodes. Chart parsers are often specially engineered for high-speed decoding. (e.g., using Cython)

Other parsers. Shen et al. (2018) and Nguyen et al. (2020) proposed local-and-greedy parsers in the top-down splitting style. Their models facilitate divide-and-conquer algorithms that construct the tree based on the magnitude of the splitting scores. A similar way of leveraging concurrent and greedy operations appears in an easy-first parser (Goldberg and Elhadad, 2010). Sequential labeling (GómezRodríguez and Vilares, 2018; Wei et al., 2020) is a new active thread that also enables parallelism and fast decoding. Collobert (2011) designed an iterative chunking process for parsing. His work stratifies trees into levels of IOBES prefixed constituent chunking nodes. Similar to ours, his parser works from the bottom levels to higher levels. However, the complexity is fixed at $O\left(n^{2}\right)$ without any node combinations. All models introduced in this section do not exploit vector compositionality. 


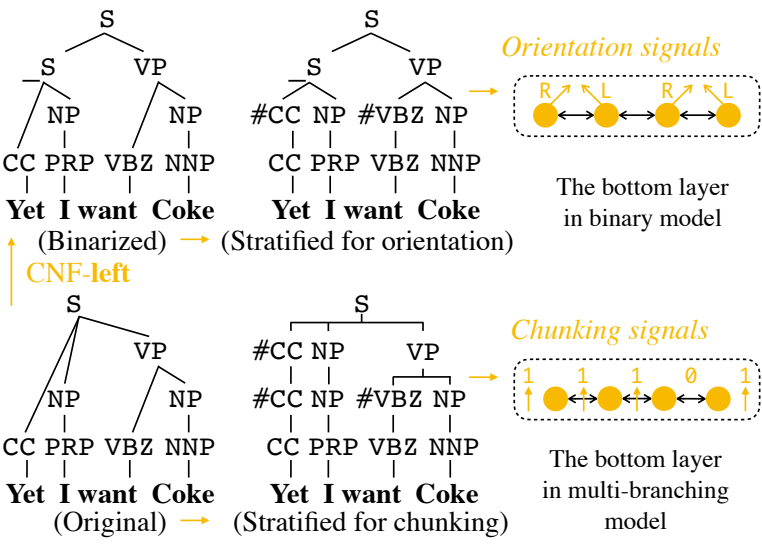

Figure 3: Example illustrating our tree stratification. Both binary and multi-branching stratified trees contain redundancy, from which the original tree can be recovered with a few simple heuristic rules. Four in a layer combine into two with a compression ratio of $1 / 2$.

\begin{tabular}{ccc}
\hline Category & Samples & \# Types \\
\hline Original & S NP VP SBAR+S & 104 \\
Sub & _S _NP _VP _PP & 25 \\
\#POS & \#NNP \#DT \# JJ \#. & 45
\end{tabular}

Table 1: Three categories of our constituent label set with their samples and number of types. This is created from Penn Treebank (PTB). '_Sub' and '\#POS' are relaying types, which we group into a sub category.

\section{Neural Combinatory Parsing}

\subsection{Data and Complexity}

Our models require stratified trees to train recurrent layers, and the binary model requires further binarization. Stratification and binarization introduce redundant relaying nodes to the trees.

Tree binarization. From the bottom-up perspective, a binary tree describes the order in which words and constituents combine with their neighbors into larger constituents, as shown in Figure 3. The orientations of the four words (i.e., right-leftright-left) determine the first combination.

After binarization, we label the relaying subconstituents with the parent label prefixed with an underscore mark. If terminal POS tags do not immediately form constituents, we create relaying placeholders prefixed with a hash mark $^{2}$, as presented in Table 1. Unary branches were collapsed into a single node. Plus marks were used to join their labels (e.g., SBAR+S), and all trace branches were removed. The CNF with either a left or a

\footnotetext{
${ }^{2}$ Multi-branching trees do not require binarization. The '_Sub' group disappears, but the '\#POS' group persists.
}

\begin{tabular}{ccccc}
\hline $\begin{array}{c}\text { CNF } \\
\text { Ori. }\end{array}$ & \multicolumn{2}{c}{ Left-factoring } & \multicolumn{2}{c}{ Right-factoring } \\
PTB & $3.8 \mathrm{M}$ & $\underline{4.4 \mathrm{M}}$ & \multicolumn{1}{c}{ Left } & Right \\
\hline CTB & $\underline{2.5 \mathrm{M}}$ & $1.7 \mathrm{M}$ & $1.4 \mathrm{M}$ & $\underline{\underline{6.5 \mathrm{M}}}$ \\
KTB & $\underline{4.5 \mathrm{M}}$ & $0.9 \mathrm{M}$ & $1.8 \mathrm{M}$ & $\underline{2.1 \mathrm{M}}$ \\
\hline nCNF & \multicolumn{2}{l}{ Midin-factoring } & \multicolumn{2}{l}{ Midout-factoring } \\
\hline PTB & $3.0 \mathrm{M}$ & $\underline{5.3 \mathrm{M}}$ & $2.8 \mathrm{M}$ & $\underline{5.2 \mathrm{M}}$ \\
CTB & $1.9 \mathrm{M}$ & $\underline{2.2 \mathrm{M}}$ & $1.7 \mathrm{M}$ & $\underline{2.1 \mathrm{M}}$ \\
KTB & $\underline{2.8 \mathrm{M}}$ & $\underline{1.7 \mathrm{M}}$ & $\underline{2.5 \mathrm{M}}$ & $1.2 \mathrm{M}$ \\
\hline
\end{tabular}

Table 2: Frequencies of orientation with different CNF (biased) and non-CNF (balanced) factors in different stratified corpora.

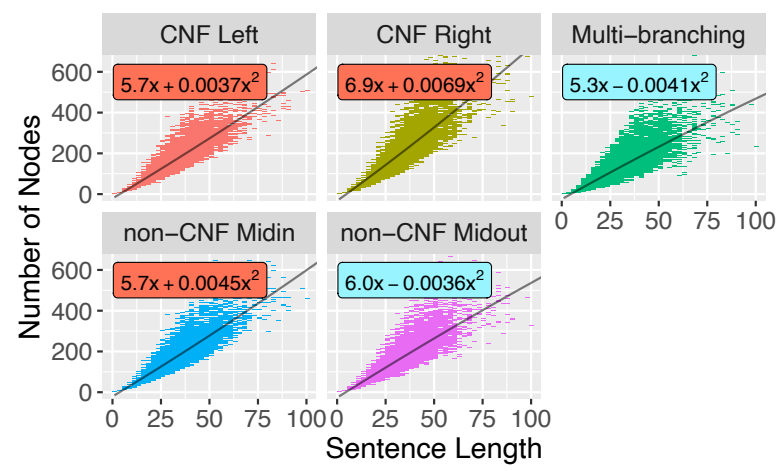

Figure 4: Linear empirical complexity in stratified PTB. Linear regression reflects insignificant $O\left(n^{2}\right)$ tendencies. We differentiated the quadratic terms with red or light blue colors and omitted the constant biases.

right factor is commonly used. However, it is heuristically biased, and trees can be binarized using other balanced splits such as always splitting from the center to create a complete binary tree (mid-out) and iteratively performing left and right to create another balanced tree (mid-in). Finally, the orientation is extracted from the paths of these binary trees.

We binarized Penn Treebank (Marcus et al., 1993, PTB) for English, Chinese Treebank (Xue et al., 2005, CTB) for Chinese, and Keyaki Treebank $^{3}$ (Butler et al., 2012, KTB) for Japanese to present the syntactic branching tendencies in Table 2. As English is a right-branching language, its majority orientation is to the right. Even leftfactoring cannot reverse the trend, but it should create a greater balance. Figure 4 shows that it is less effective to stratify PTB with a right factor because it enhances the tendency. The reverse tendency emerges in the KTB corpus as Japanese is a left-branching language. For Chinese, CTB does

\footnotetext{
${ }^{3}$ https: / / github.com/a jb129/ KeyakiTreebank/tree/master/treebank
} 


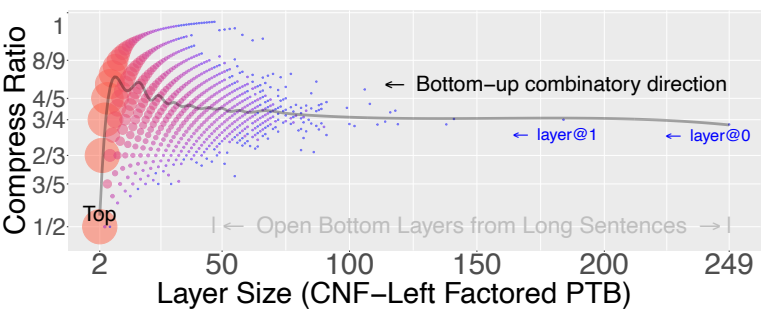

Figure 5: Layer-wise compression ratio over different sizes. The dot size was proportional to the situation count. Statistically, the ratio had a stable mean of 0.77 .

not exhibit a clear branching tendency. Non-CNF factors preserve the original tendency.

Complexity. Our models are trained with stratified treebanks. The complexity for inference follows the total number of nodes in each layer of a tree. There are two ideal cases: 1) Complete balanced trees with complexity $O(n)$. They contain multiple independent phrases and enable full concurrency. 2) Trees with a single dependency core. The model reduces a constant number of nodes in each layer, resulting in $O\left(n^{2}\right)$ complexity.

While each parse is a mixture of many cases, the empirical complexity prefers the first case. Formally, the average-case complexity can be inferred as $O(n)$ with the help of a stable compression ratio $0<C<1\left(C \geq 0.5\right.$ for binary). Let $m_{i}$ represent the number children of the $i$-th tree in a general layer; the compression ratio can be stated as $C=\frac{\sum_{i} 1}{\sum_{i} m_{i}}$. Our stratified treebanks give stable $C \mathrm{~s}$ for layers of different lengths, as shown in Figure 5. For the $k$-th layer of a sentence with $n$ words, the number of nodes to compute can be expected to be $C^{k} \cdot n$. Based on tree height $K>0$, the expected number of total parsing nodes is

$$
\sum_{k=0}^{K} C^{k} \cdot n=n \cdot \sum_{k=0}^{\infty} C^{k}-n \cdot \sum_{k=K+1}^{\infty} C^{k}<\frac{n}{1-C} .
$$

The partial geometric series determines an empirically linear complexity on average.

Theoretically, the complexity has a quadratic upper bound. The general layer with

$$
m_{i}= \begin{cases}M & \text { if } i=j \\ 1 & \text { otherwise }\end{cases}
$$

entails the second case, where $m_{j}$ is the only $M$ ary branch in each layer. The nodes shape a triangular stratified tree with an $O\left(n^{2}\right)$ complexity. However, this case is rare, especially for long sentences

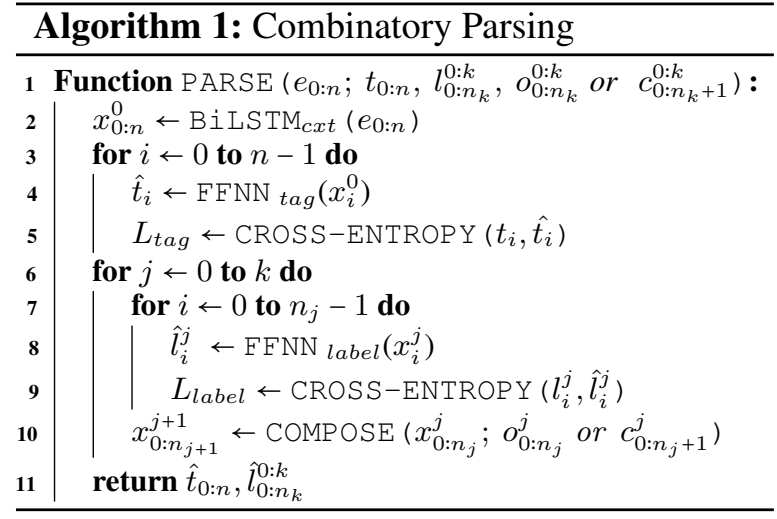

that should contain several concurrent phrases. Otherwise, regression in Figure 4 should show significant $O\left(n^{2}\right)$ tendencies. (See Appendix A.1 for more support and examples.)

Data structure. To summarize the data components of a treebank corpus, we used four tensors of indices for 1) words, 2) POS tags, 3) stratified syntactic labels, 4) stratified orientations, or 5) stratified chunks,

$$
\left(x_{0: n}, t_{0: n}, l_{0: n_{k}}^{0: k}, o_{0: n_{k}}^{0: k} \text { or } c_{0: n_{k}+1}^{0: k}\right)_{j} \in D
$$

where $n$ is the length of the $j$-th sentence, $k$ indicates the $k$-th layer of the stratified data, and $n_{k}$ is the layer length. ":" indicates a range of a sequence.

\subsection{Combinatory Parsing}

Our models comprise four feedforward (FFNN) and two bidirectional LSTM (BiLSTM) networks to decompose parsing into collaborative functions, as shown in Algorithm 1. During training, we use teacher forcing. In the inference phase, the supervised signals behind all semicolons are ignored; the predicted signals serve as their substitute.

Input $e_{0: n}$ is an embedding sequence indexed by $x_{0: n}$. In lines 2-5, the model prepares a contextual sequence for the combinator and predicts the lexical tags. Lines 6-10 describe the layer-wise loop of the combinator.

The tagging and labeling functions, $\mathrm{FFNN}_{t a g}$ and $\mathrm{FFNN}_{\text {label }}$, are 2-layer FFNNs. Their first layer is shared, creating a hidden layer necessary for projecting diversified situations in the manifold to the non-zero logits for the argmax decision. The core function $\mathrm{COMPOSE}^{4}$ is either a binary Algorithm 2 or a multi-branching Algorithm 3.

\footnotetext{
${ }^{4}$ COMPOSE with BiLSTM cannot be parallelized to $O(1)$.
} 

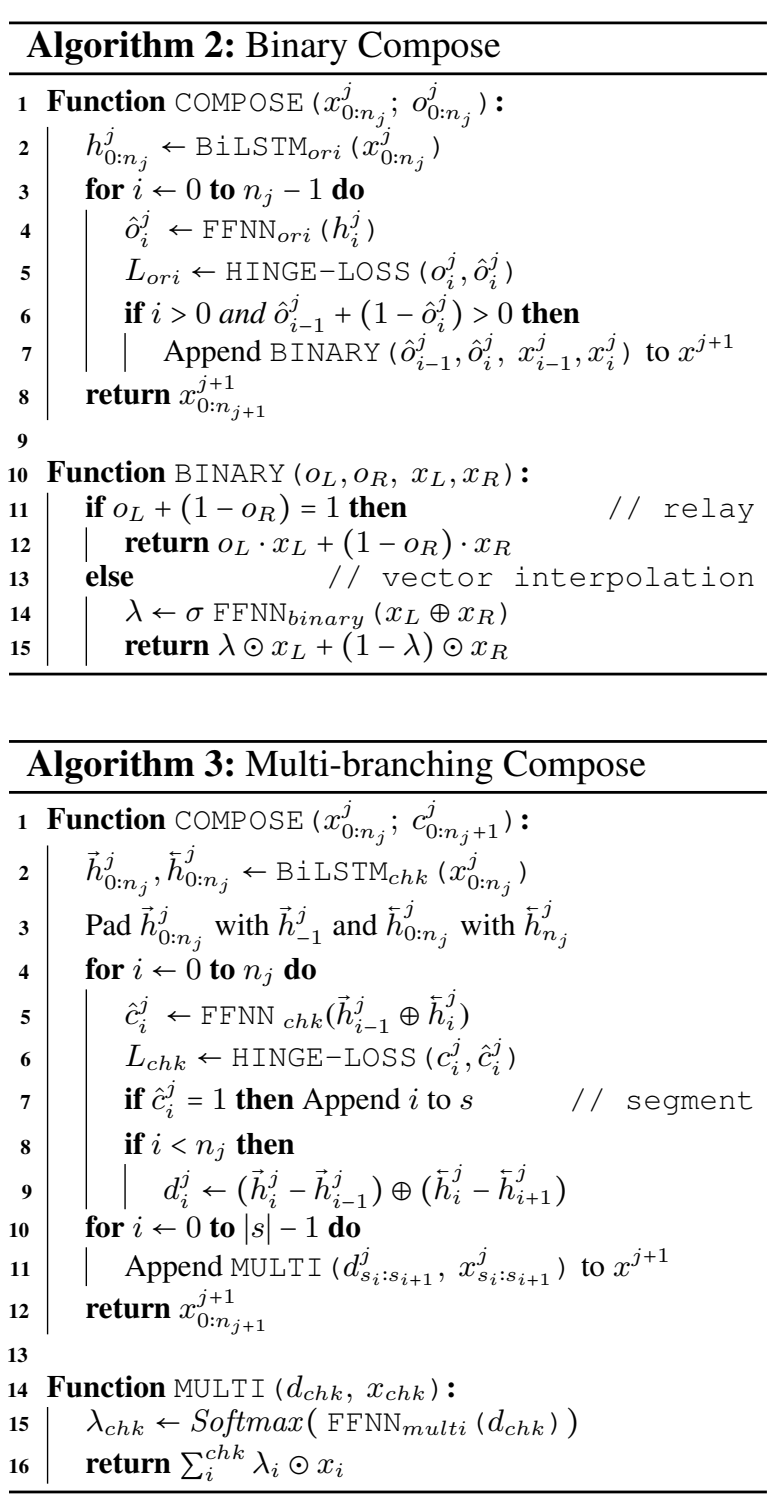

Binary model. In Algorithm 2, the orientation function is hinted by BiLSTM ori $_{\text {. A single-layer }}$ FFNN $_{\text {ori } i}$ with a threshold reduces the outputs to an integer of either 0 or 1 to indicate two possible orientations. In function BINARY, when two adjacent orientations agree as they sum to 2 , their embeddings are combined by a combinatory operation. $\sigma$ is the Sigmoid function, " $\oplus$ " represents concatenation, and " $\odot$ " represents pointwise multiplication. (See Appendix A.3 for more binary variants.)

Multi-branching model. To resemble binary interpolation, we use the Softmax function for each chunk, as described in Algorithm 3. BiLSTM is in place of $\mathrm{BiLSTM}_{\text {ori }}$ to hint $\mathrm{FFNN}_{c h k}$ emitting chunk signals. Segment $s$ splits $x_{0: n_{j}}^{j}$ and $d_{0: n_{j}}^{j}$ into chunks of $x_{c h k}$ and $d_{c h k}$. FFNN multi $_{\text {and Softmax }}$ turn $d_{c h k}$ into attention $\lambda_{c h k}$ to interpolate vector chunk $x_{c h k}$. Binary interpolation $\lambda$ is a special case of the multi-branching $\lambda_{c h k}$ because Sigmoid and Softmax functions are closely related.

To obtain the final tree representation, we apply a symbolic pruner in the same bottom-up manner to remove redundant nodes, expand the collapsed nodes, and assemble the sub-trees based on the neural outputs. (See Appendix A.4.)

\section{Experiments}

We follow previous data splits for PTB, CTB, and KTB (See Appendix A.2). The preprocessing of data is described in Section 3.1.

For the binary model, we explored interpolated dynamic datasets by sampling two CNF factored datasets. This is because of the following: 1) The experiments with the non-CNF factors did not yield any promising results; thus, we have not reported them. 2) The language was loosely left-branched, right-branched, or did not show a noticeable tendency. Moreover, the use of a single static dataset may introduce a severe orientation bias. 3) All factors are intermediate variables and equally correct. We defined the sampling strategies with two static CNF-factored datasets at certain ratios and named each strategy in the format "L\%R\%" according to the ratio percentages. Our experiments mainly focus on binary model $\mathbf{B}$ because of the aforementioned property for training parsers more accurate than multi-branching model $\mathbf{M}$.

Our parsers do not contain lexical information components (Liu and Zhang, 2017c; Kitaev and Klein, 2018). Instead, we use fastText (Bojanowski et al., 2017) because we can obtain pre-trained models easily for many languages or train new ones from scratch with the corpora at hand. We examined its influence in Section 4.2, whereas the official pre-trained embeddings are the default.

Meanwhile, pre-trained language models are useful for various tasks, including constituency parsing (Kitaev and Klein, 2018, 2020; Zhou and Zhao, 2019; Yang and Deng, 2020; Mrini et al., 2020). We chose XLNet (Yang et al., 2019) to compare with the static fastText embeddings. Specifically, either a 1-layer FFNN (/0) or an $n$-layer BiLSTM $\left(/ n^{+}\right)$was used to convert the 768-unit output to our model size. We used a GeForce GTX 1080 Ti with 11 GB and a TITAN RTX with 24GB memory only for tuning XLNet.

The model size for vector compositionality was set at 300. The hidden sizes for labeling, orientation, and chunking were 200,64 , and 200 , re- 


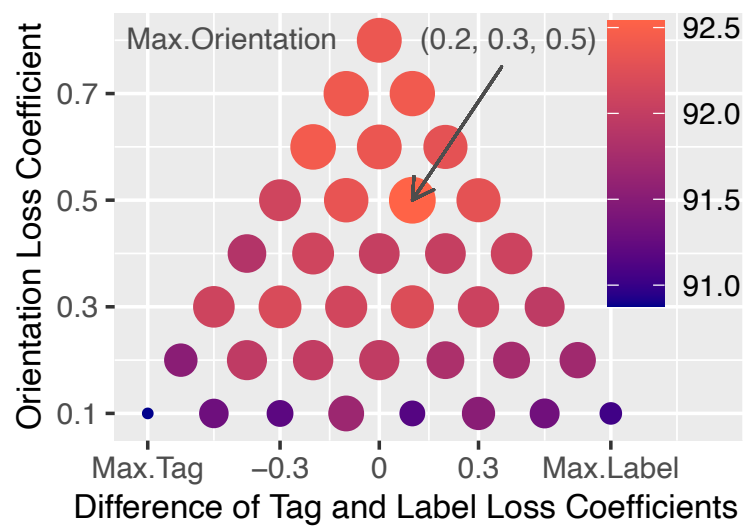

Figure 6: Grid search with an interval of 0.1 in a space of (tag, label, orientation) loss coefficients. The best was $(0.2,0.3,0.5)$ indicated by an arrow.

spectively. Different numbers of layers of the $\operatorname{BiLSTM}_{c x t}(/ n)$ were explored, and the default was six layers. HINGE-LOSS was the default criterion for orientation while binary cross-entropy (BCE-LOSS) was tested. The coefficients of the three losses were explored and the default were $L=0.2 \cdot L_{\text {tag }}+0.3 \cdot L_{\text {label }}+0.5 \cdot L_{\text {ori }}($ or chk $)$.

\subsection{Overall Results}

Table 3 lists the parsing accuracies and speeds of the single models in ascending order according to their F1 scores for the PTB corpus. The transitionbased parsers with $O(n)$ complexity appear at the top of the table, followed by other types of models, and the chart parsers running in $O\left(n^{3}\right)$ time are at the bottom of the table. The models exhibited similar trends for the CTB. Shen et al. (2018) and our models belong to type $\mathrm{O}$ and have similar complexities. Generally, the accuracy follows the complexity, whereas the speed roughly follows the year of publication rather than complexity or type.

\subsection{Comparison of Models}

Models with fastText. We investigated the binary model through ablation. The impacts of fastText are presented in the upper part of Table 4. B/E does not require any external data beyond PTB, which is comparable to models without a pre-trained GloVe (Pennington et al., 2014).

Then, we replaced BiLSTM ori $_{i}$ with an FFNN to examine its effect. The results are in the bottom rows. The comparison proves whether the embeddings are collaborative for the orientation signals because FFNN regards each input independently.

Finally, we used a grid search to explore the hyperparameter space of our three-loss coefficients.

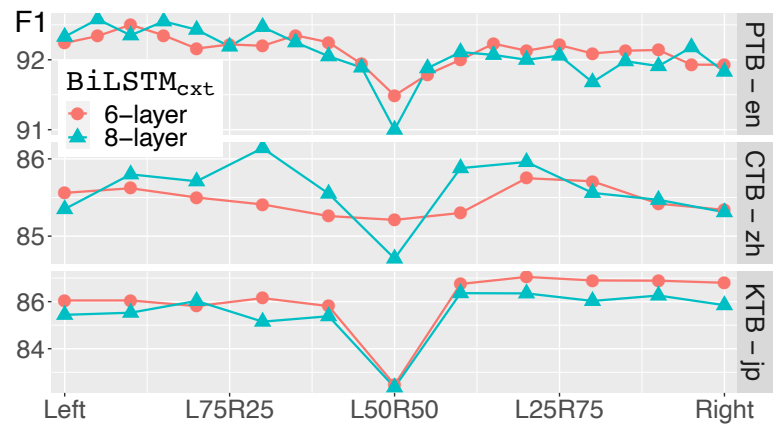

Figure 7: Probabilistic interpolations of two CNF fac-

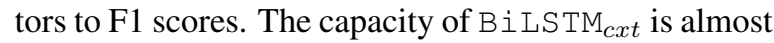
saturated with 6 or 8 layers.

Figure 6 shows that the performance correlates to the orientation loss the most, but it is not overly sensitive to the hyperparameters.

Pre-trained language model. We compared the results using frozen fastText with those using frozen $\mathrm{XLNet}^{5}$ in Table 5. The accuracy of the model increased along with the depth of BiLSTM $_{c x t}$, and it exhibited the most significant increase across all variants. Owing to XLNet, our complexities grew to $O\left(n^{2}\right)$.

We fine-tuned our models ${ }^{6}$ and compared them with other parsers using fine-tuned language models. These are listed in Table 6.

\subsection{Tree-Binarization Strategy}

To reflect the branching tendency, our best single model for PTB was obtained on the dynamic L95R05 dataset. This dataset is a probabilistic interpolation between the left-factored dataset (for 95\% chances) and a right-factored dataset (for 5\% chances) in Figure 7. The best model for CTB appeared on the left side at L70R30, scoring 86.14, whereas the best for KTB was on the L30R70 dataset, scoring 87.05 with a 6-layer BiLSTM C $_{c x t}$. Typically, the results for all the corpora had a minimum at L50R50. For English, the left "wing" was higher than the right; the opposite trend was observed for Japanese. For Chinese, no clear trend was obtained.

All studies described in the previous sections were conducted on the PTB L85R15 dataset.

\footnotetext{
${ }^{5}$ XLNet tokenizes words into sub-word fractions. For the frozen XLNet, using leftmost, rightmost, or averaged subword embeddings as the word input yielded similar results.

${ }^{6}$ For the fine-tuned XLNet, using either the leftmost or rightmost sub-word yielded similar results earlier. However, averaging sub-words produced F1 scores under 94.
} 


\begin{tabular}{l|lcccc|cccc}
\hline Corpus & \multicolumn{4}{|c|}{ Penn Treebank } & \multicolumn{4}{c}{ Chinese Treebank } \\
Single Model & Type & sents/sec & LP & LR & F1 & Type & LP & LR & F1 \\
\hline Watanabe and Sumita (2015) & $\mathrm{T} \uparrow(32)$ & - & - & - & 90.7 & $\mathrm{~T} \uparrow(64)$ & - & - & 84.3 \\
Gómez-Rodríguez and Vilares (2018) & $\mathrm{O}$ & $\underline{898}$ & - & - & 90.7 & $\mathrm{O}$ & - & - & 83.1 \\
Cross and Huang (2016) & $\mathrm{T} \uparrow(1)$ & - & 92.1 & 90.5 & 91.3 & - & - & - & - \\
Liu and Zhang (2017c) & $\mathrm{T} \downarrow(16)$ & 79.2 & 92.1 & 91.3 & 91.7 & $\mathrm{~T} \downarrow(16)$ & 85.9 & 85.2 & 85.5 \\
Stern et al. (2017) & $\mathrm{C}$ & 75.5 & 93.0 & 90.6 & 91.8 & - & - & - & - \\
Shen et al. (2018) & $\mathrm{O} \downarrow(1)$ & 111.1 & 92.0 & 91.7 & 91.8 & $\mathrm{O} \downarrow(1)$ & 86.6 & 86.4 & 86.5 \\
Charniak and Johnson (2005) & $\mathrm{C}$ & - & - & - & 92.1 & - & - & - & - \\
Ours (multi-branching) & $\mathrm{O} \uparrow(1)$ & $\underline{1122.6}$ & 92.1 & 92.1 & 92.1 & $\mathrm{O} \uparrow(1)$ & 86.0 & 84.7 & 85.3 \\
Ours (binary) & $\mathrm{O} \uparrow(1)$ & $\mathbf{1 3 2 7 . 2}$ & $\mathbf{9 2 . 8}$ & $\mathbf{9 2 . 3}$ & $\mathbf{9 2 . 5}$ & $\mathrm{O} \uparrow(1)$ & 85.8 & 86.2 & 86.0 \\
Nguyen et al. (2020) & $\mathrm{O} \downarrow(1)$ & 130.2 & 92.8 & 92.8 & 92.8 & - & - & - & - \\
Kitaev and Klein (2018) & $\mathrm{C}$ & 212.5 & 93.9 & 93.2 & 93.6 & $\mathrm{C}$ & 91.9 & 91.5 & 91.7 \\
Wei et al. (2020) & $\mathrm{O} \downarrow(1)$ & 155 & 94.1 & 93.3 & 93.7 & $\mathrm{O} \downarrow(1)$ & 89.9 & 87.4 & 88.7 \\
Zhou and Zhao (2019) & $\mathrm{C}$ & 226.3 & 93.9 & 93.6 & 93.7 & $\mathrm{C}$ & 92.3 & 92.0 & 92.2 \\
Zhang et al. (2020) & $\mathrm{C}$ & 1092 & 94.2 & 94.0 & 94.1 & $\mathrm{C}$ & 89.7 & 89.9 & 89.8 \\
\hline
\end{tabular}

Table 3: Single-model results on PTB and CTB test datasets sorted by the F1 scores on PTB. Transition-based parsers, chart parser, and others are marked as T, C, and O, respectively; $\uparrow$ and $\downarrow$ denote bottom-up and top-down. The number in brackets indicates the beam size. Kitaev and Klein (2018) used Tesla K80, and the CTB scores are cited from Kitaev et al. (2019). Zhou and Zhao (2019) used GeForce GTX 1080 Ti (same condition).

\begin{tabular}{llc}
\hline Var & \multicolumn{1}{c}{ Specification } & F1 \\
\hline B/e & without fastText initialization. & 91.73 \\
B/ $\epsilon$ & with tuned official fastText. & 91.69 \\
B/E & with frozen fastText from PTB. & 92.31 \\
\hline B/F & BiLSTM $_{\text {ori }}$ into FENN & $\star$ \\
Bri $i$ & 88.97 \\
B/L & BiLSTM $_{\text {ori }}$ with BCE-LOSS. & 92.32 \\
\hline
\end{tabular}

Table 4: Results of ablation studies on fastText (top)

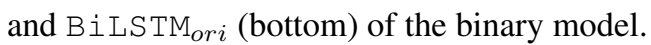

\begin{tabular}{c|cc|cc}
\hline & \multicolumn{2}{|c|}{ Frozen fastText } & \multicolumn{2}{c}{ Frozen XLNet } \\
Var & F1 & sents/sec & F1 & sents/sec \\
\hline B/0 & 65.02 & $\underline{1386.6}$ & 89.24 & $\underline{411.2}$ \\
B/2 & 91.34 & 1350.0 & 93.74 & 398.4 \\
B/6 & $\underline{92.54}$ & 1327.2 & $\underline{93.89}$ & 382.7 \\
\hline
\end{tabular}

Table 5: Effectiveness of using frozen static word embeddings or dynamic sub-word language model and corresponding peak speed.

\subsection{Complexity and Speed}

To test our linear speed advantage, we inflated our training data with redundant nodes to resemble the triangular chart of CYK algorithm, as depicted in Figure 8 and Table 7. The parse in the triangular treebank has the worst-case complexity of $O\left(n^{2}\right)$. Meanwhile, training with linearity halved the training time, reduced memory usage, and canceled the length limit for our three corpora. There is a sheer difference between linearity and squared complexity.

\begin{tabular}{l|ccc}
\hline Fine-Tuned Model & F1 & sents/sec & Type \\
\hline Kitaev and Klein (2018) & 95.13 & 70.8 & $\mathrm{C}$ \\
Kitaev and Klein (2020) & 95.44 & $\underline{1200}$ & $\mathrm{~T}$ \\
Nguyen et al. (2020) & 95.48 & - & $\mathrm{O} \downarrow$ \\
Zhang et al. (2020) & 95.69 & - & $\mathrm{C}$ \\
Wei et al. (2020) & 95.8 & - & $\mathrm{O} \downarrow$ \\
Zhou and Zhao (2019) & 96.33 & 64.8 & $\mathrm{C}$ \\
Yang and Deng (2020) & 96.34 & 71.3 & $\mathrm{~T}$ \\
Mrini et al. (2020) & $\underline{96.38}$ & 59.2 & $\mathrm{C}$ \\
\hline B/0 (XLNet+FFNN) & $\underline{95.72}$ & $\underline{411.2}$ & $\mathrm{O} \uparrow$ \\
B/2 (XLNet+BiLSTM) & 94.67 & 398.4 & $\mathrm{O} \uparrow$ \\
M/0 (XLNet+FFNN) & 95.44 & 369.4 & $\mathrm{O} \uparrow$ \\
\hline
\end{tabular}

Table 6: Improvements with pre-trained language models. We used a greedy search algorithm on single GeForce GTX 1080 Ti. Rows 6-8 are reported by Yang and Deng (2020) using GeForce GTX 2080 Ti. Kitaev and Klein (2020) used a cloud TPU with a beam search algorithm and a larger batch size.

\section{Discussion}

\subsection{Model Structure}

Our parsers comprise a neural encoder for scoring (i.e., Algorithm 1) and a non-neural decoder for searching. The decoder is a symbolic extension of the encoder in that both run in bottom-up manner, and the decoder interprets the scores as local-andgreedy decisions. Other neural parsers also fit a similar encoder-decoder framework. However, decoders with dynamic programming often include forward and backward processes heterogeneous to 


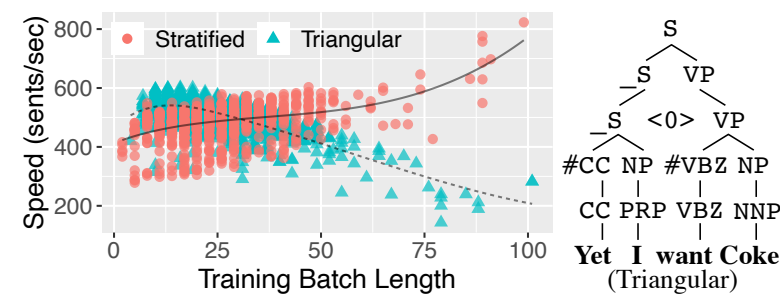

Figure 8: Linear complexity vs. squared complexity. Redundancy with placeholder " $<0>$ " helps maintain the triangular shape.

\begin{tabular}{c|ccc}
\hline Format & Time/150 & Memory & OOM \\
\hline Stratified & 7.5 hours & $3.3 \mathrm{~GB}$ & - \\
Triangular & 15.9 hours & $8.2 \mathrm{~GB}$ & 100 \\
\hline
\end{tabular}

Table 7: Training time and memory consumed by our two data formats. The time column indicates the time used for 150 training epochs with validations. Development F1 scores are approximately 92.4. The OOM column lists the length limit for preventing an out-ofmemory error. Kitaev and Klein (2018) took 10 hours for 93 training epochs on our GeForce GTX 1080 Ti to yield their results.

their forward encoders (Kitaev and Klein, 2018, 2020). The encoder and decoder in our model and Shen et al. (2018) are more homogeneous and can be easily merged. Our parsers are bottom-up combinatory, while theirs was top-down splitting. Similar homogeneity can be found in an easy-first dependency parser (Goldberg and Elhadad, 2010).

Input component. In terms of encoder, Tables

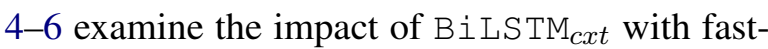
Text or XLNet, and the following conclusions can be drawn. 1) The top rows of Table 4 suggest that frozen fastText embeddings contain sub-word information, whereas tuning them disturbs the frozen information because the n-gram model is not part of our model. 2) Table 5 shows that the deeper

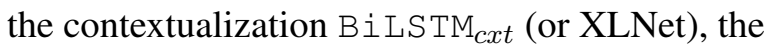
better the results. 3) Tables 5 and 6 indicate that the tuning process for the pre-trained language models (Peters et al., 2018; Devlin et al., 2019; Yang et al., 2019) achieves a significant improvement.

Speed and size. One of our research goals was to achieve simplicity and efficiency. In terms of speed, our models parallelize more actions than transition-based parsers and have fewer computing nodes than chart parsers. In terms of size, our models contain approximately $4 \mathrm{M}$ parameters in addition to the $13 \mathrm{M}$ fastText (or 114M XLNet) pre-trained embeddings, which is fewer than those

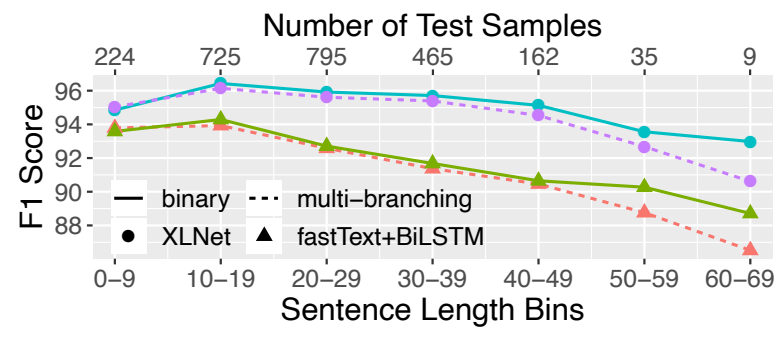

Figure 9: XLNet provides an overall improvement for all models and length bins. All models find it challenging to handle long sentences.

of Shen et al. $(2018,22 \mathrm{M}+)$ and Kitaev and Klein $(2018,26 \mathrm{M})$. The recursiveness and productiveness of vector compositionality should account for the compact size.

Vector compositionality. The performance of $\mathrm{FFNN}_{\text {ori }}^{\star}$ is inferior to that of its RNN counterparts, suggesting that some information might not be encoded locally. Thus, the COMPOSE function should remain in a contextual form to collaboratively leverage the whole layer. However, BiRNN might still be a bottleneck for long-range orientation, as sug-

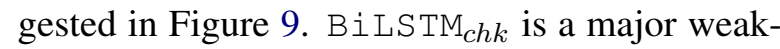
ness of the multi-branching model, especially for longer sentences.

\subsection{Tree Binarization and Headedness}

Tree binarization. Probabilistic interpolation with two CNF-factored datasets is effective for the three languages studied, as shown in Figure 7. Dynamic sampling allows the model to cover a wider range of composed vectors to improve its robustness to ambiguous orientations. Furthermore, it seems counterintuitive for human learners to obtain the best model using left-biased interpolation for a right-branching language or vice versa. However, for a neural model, balancing the frequency seems to be the key factor for improving performance (Sennrich et al., 2016; Zhao et al., 2018). The fact that the L50R50 dataset yielded the worst models also suggests that the balance should be based on a default orientation tendency. This could also be the reason why mid-in or mid-out did not improve the model.

Headedness. Figure 10 show the intermediate parses on the same sentence from our two models. They are typical examples in the output.

The binary model first combines determiners and their right neighbors rather than adjectives and nouns in noun phrases (blue spans). It also post- 

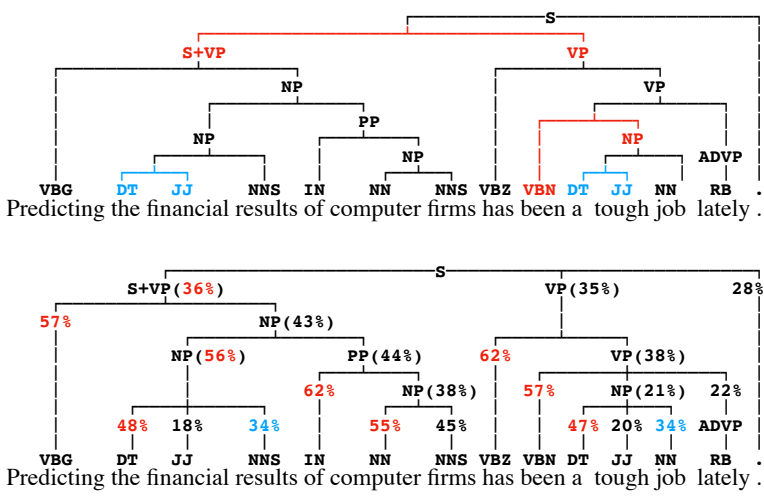

Figure 10: English internal constituents (top) and headedness (bottom) from our two models.

\begin{tabular}{|c|c|}
\hline Parent (\#) & "Head child by maximum weight \\
\hline $\begin{array}{ll}\mathrm{NP} & (14.4 \mathrm{~K})\end{array}$ & $\begin{array}{l}\text { DT }(4.5 \mathrm{~K}) ; \star \operatorname{NP}(4.3 \mathrm{~K}) ; \star \operatorname{NNP}(1.6 \mathrm{~K}) ; \\
\star \mathrm{JJ}(922) ; \star \mathrm{NN}(751) ; \star \operatorname{NNS}(616) ; \\
\text { etc. }(1.6 \mathrm{~K} ; 38 \text { of } 50 \text { types with “*”) }\end{array}$ \\
\hline VP $\quad(6.8 \mathrm{~K})$ & $\begin{array}{l}\text { VBD (1.5K); VB (1.4K); VBZ (1.0K); } \\
\text { VBN (954); VBP ( } 705) ; \text { MD (523); } \\
\text { VBG (387); VP (169); TO (81); etc. }\end{array}$ \\
\hline$(5.5 \mathrm{~K})$ & IN (5.0K); TO (397); etc. \\
\hline$(3.8 \mathrm{~K})$ & VP (3.4K); S (194); NP (90); etc. \\
\hline $\operatorname{SBAR}(1.2 \mathrm{~K})$ & $\begin{array}{l}\text { IN (649); WHNP (395); WHPP (19); } \\
\text { WHADVP (121); SBAR (15); etc. }\end{array}$ \\
\hline ADVP (278) & RB (181); IN (30); RBR (25); etc. \\
\hline (198) & CD (67); IN (65); RB (29); JJR (16); \\
\hline
\end{tabular}

Table 8: English headedness selection with our multibranching model on PTB test set. " $\star$ " marks the absence of a DT child for its NP sisters. For quantifier phrases ( $Q P)$, some non-quantifiers are more likely to be heads if they appear; e.g., adverbs (RB; e.g., "approximately"), prepositions (IN; e.g., "about"), and relative adjectives (JJR; e.g., "more than").

pones the combination with adjuncts such as punctuation and adverb (red spans). The high frequencies of determiners in noun phrases make them great attractors.

On the other hand, the multi-branching model places close attention on what the syntactic head is supposed to be. In the noun phrases, determiners receive the highest weight averages (red), and the nouns obtain the second (blue). This phenomenon suggests that an English noun phrase's syntactic role is mainly projected from the determiners, as discussed by Zwicky (1985). Table 8 provides more statistical support. For example, the model selects DT as an NP head if it is available; otherwise, nouns and adjectives are prominent heads. Chinese and Japanese parsers work similarly for their headedness. (See Appendix A.5.)

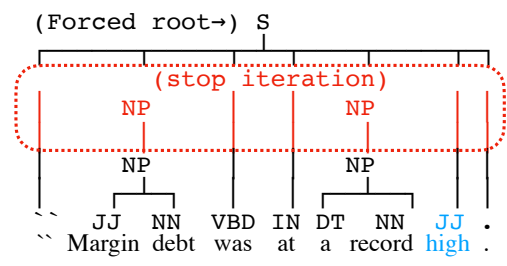

Figure 11: Failed parse from the multi-branching model. The model stops parsing and saves computations when it repeats the same chunking positions.

\subsection{Error Analysis}

The rate of an invalid parse is the last topic that we consider for our parsers. For the binary parser, fatal errors, such as frame-breaking orientations, appear at an early stage of training. However, the late $90 \%$ of training time contains very few errors, and our binary model is free from invalid parsing on the test set. For the multi-branching parser, it is observed that 11 out of 2,416 test parses are forests rather than parse trees when they are trained with fastText. However, the multi-branching parser with fine-tuned XLNet reduces the error count on the test set to 1 .

We present a failed multi-branching parse with fastText, as shown in Figure 11. The postnominal adjective "high" is uncommon for English. Because the model did not group it with the adjacent "a record" to form an NP, the error propagated to higher layers (e.g., no PP as an adjunct to form a VP), causing the bad parse. It implies that the multibranching model requires an appropriate predictargument configuration to chunk.

\section{Conclusion}

We proposed a pair of neural combinatory constituency parsers. The binary one yields F1 scores comparable to those of recent neural parsers. The multi-branching one reveals constituency headedness. Both are simple and efficient with relatively high speeds. We also leveraged a pre-trained language model and CNF factors to increase the accuracy. We reflected the branching tendencies of three languages.

\section{Acknowledgments}

We extend special thanks to our reviewers for invaluable comments and Hayahide Yamagishi for initial discussions. This work has been partly supported by the Grant-in-Aid for Scientific Research from the Japan Society for the Promotion of Science (JSPS KAKENHI); Grant Number 19K12099. 


\section{References}

Dzmitry Bahdanau, Kyunghyun Cho, and Yoshua Bengio. 2015. Neural Machine Translation by Jointly Learning to Align and Translate. In Proceedings of the 3rd International Conference on Learning Representations.

Piotr Bojanowski, Edouard Grave, Armand Joulin, and Tomas Mikolov. 2017. Enriching Word Vectors with Subword Information. Transactions of the Association for Computational Linguistics, 5:135-146.

Alastair Butler, Tomoko Hotta, Ruriko Otomo, Kei Yoshimoto, Zhen Zhou, and Hong Zhu. 2012. Keyaki Treebank: phrase structure with functional information for Japanese. In Proceedings of Text Annotation Workshop, 2012.

Eugene Charniak. 2000. A Maximum-EntropyInspired Parser. In Proceedings of 6th Applied Natural Language Processing Conference, pages 132139.

Eugene Charniak and Mark Johnson. 2005. Coarseto-Fine n-Best Parsing and MaxEnt Discriminative Reranking. In Proceedings of the Conference of the 43rd Annual Meeting of the Association for Computational Linguistics, pages 173-180.

Ronan Collobert. 2011. Deep Learning for Efficient Discriminative Parsing. In Proceedings of the Fourteenth International Conference on Artificial Intelligence and Statistics, volume 15 of JMLR Proceedings, pages 224-232.

James Cross and Liang Huang. 2016. Span-Based Constituency Parsing with a Structure-Label System and Provably Optimal Dynamic Oracles. In Proceedings of the 2016 Conference on Empirical Methods in Natural Language Processing, pages 1-11.

Jacob Devlin, Ming-Wei Chang, Kenton Lee, and Kristina Toutanova. 2019. BERT: Pre-training of Deep Bidirectional Transformers for Language Understanding. In Proceedings of the 2019 Conference of the North American Chapter of the Association for Computational Linguistics: Human Language Technologies, pages 4171-4186.

Chris Dyer, Adhiguna Kuncoro, Miguel Ballesteros, and Noah A. Smith. 2016. Recurrent Neural Network Grammars. In Proceedings of the 2016 Conference of the North American Chapter of the Association for Computational Linguistics: Human Language Technologies, pages 199-209.

Yoav Goldberg and Michael Elhadad. 2010. An Efficient Algorithm for Easy-First Non-Directional Dependency Parsing. In Human Language Technologies: Conference of the North American Chapter of the Association of Computational Linguistics, pages $742-750$
Carlos Gómez-Rodríguez and David Vilares. 2018. Constituent Parsing as Sequence Labeling. In Proceedings of the 2018 Conference on Empirical Methods in Natural Language Processing, pages 1314 1324.

Nikita Kitaev, Steven Cao, and Dan Klein. 2019. Multilingual Constituency Parsing with Self-Attention and Pre-Training. In Proceedings of the 57th Conference of the Association for Computational Linguistics, pages 3499-3505.

Nikita Kitaev and Dan Klein. 2018. Constituency Parsing with a Self-Attentive Encoder. In Proceedings of the 56th Annual Meeting of the Association for Computational Linguistics, pages 2676-2686.

Nikita Kitaev and Dan Klein. 2020. Tetra-Tagging: Word-Synchronous Parsing with Linear-Time Inference. In Proceedings of the 58th Annual Meeting of the Association for Computational Linguistics, pages 6255-6261.

Dan Klein and Christopher D. Manning. 2003. Accurate Unlexicalized Parsing. In Proceedings of the 41st Annual Meeting of the Association for Computational Linguistics, pages 423-430.

Marco Kuhlmann, Carlos Gómez-Rodríguez, and Giorgio Satta. 2011. Dynamic Programming Algorithms for Transition-Based Dependency Parsers. In Proceedings of the 49th Conference of the Association for Computational Linguistics, pages 673-682.

Jiangming Liu and Yue Zhang. 2017a. EncoderDecoder Shift-Reduce Syntactic Parsing. In Proceedings of the 15th International Conference on Parsing Technologies, pages 105-114.

Jiangming Liu and Yue Zhang. 2017b. In-Order Transition-based Constituent Parsing. Transactions of the Association for Computational Linguistics, $5: 413-424$.

Jiangming Liu and Yue Zhang. 2017c. Shift-Reduce Constituent Parsing with Neural Lookahead Features. Transactions of the Association for Computational Linguistics, 5:45-58.

Mitchell P. Marcus, Beatrice Santorini, and Mary Ann Marcinkiewicz. 1993. Building a Large Annotated Corpus of English: The Penn Treebank. Computational Linguistics, 19(2):313-330.

Tomas Mikolov, Ilya Sutskever, Kai Chen, Gregory S. Corrado, and Jeffrey Dean. 2013. Distributed Representations of Words and Phrases and their Compositionality. In Advances in Neural Information Processing Systems 26, pages 3111-3119.

Khalil Mrini, Franck Dernoncourt, Quan Hung Tran, Trung Bui, Walter Chang, and Ndapa Nakashole. 2020. Rethinking Self-Attention: Towards Interpretability in Neural Parsing. In Proceedings of the 2020 Conference on Empirical Methods in Natural Language Processing, pages 731-742. 
Thanh-Tung Nguyen, Xuan-Phi Nguyen, Shafiq R. Joty, and Xiaoli Li. 2020. Efficient Constituency Parsing by Pointing. In Proceedings of the 58th Annual Meeting of the Association for Computational Linguistics, pages 3284-3294.

Jeffrey Pennington, Richard Socher, and Christopher D. Manning. 2014. GloVe: Global Vectors for Word Representation. In Proceedings of the 2014 Conference on Empirical Methods in Natural Language Processing, pages 1532-1543.

Matthew E. Peters, Mark Neumann, Mohit Iyyer, Matt Gardner, Christopher Clark, Kenton Lee, and Luke Zettlemoyer. 2018. Deep Contextualized Word Representations. In Proceedings of the 2018 Conference of the North American Chapter of the Association for Computational Linguistics: Human Language Technologies, pages 2227-2237.

Slav Petrov, Leon Barrett, Romain Thibaux, and Dan Klein. 2006. Learning Accurate, Compact, and Interpretable Tree Annotation. In Proceedings of the 21st International Conference on Computational Linguistics and 44th Annual Meeting of the Association for Computational Linguistics, pages 433440.

Rico Sennrich, Barry Haddow, and Alexandra Birch. 2016. Neural Machine Translation of Rare Words with Subword Units. In Proceedings of the 54th Annual Meeting of the Association for Computational Linguistics, pages 1715-1725.

Yikang Shen, Zhouhan Lin, Athul Paul Jacob, Alessandro Sordoni, Aaron C. Courville, and Yoshua Bengio. 2018. Straight to the Tree: Constituency Parsing with Neural Syntactic Distance. In Proceedings of the 56th Annual Meeting of the Association for Computational Linguistics, pages 1171-1180.

Richard Socher, John Bauer, Christopher D. Manning, and Andrew Y. Ng. 2013. Parsing with Compositional Vector Grammars. In Proceedings of the 51st Annual Meeting of the Association for Computational Linguistics, pages 455-465.

Mitchell Stern, Jacob Andreas, and Dan Klein. 2017. A Minimal Span-Based Neural Constituency Parser. In Proceedings of the 55th Annual Meeting of the Association for Computational Linguistics, pages 818 827.

Oriol Vinyals, Lukasz Kaiser, Terry Koo, Slav Petrov, Ilya Sutskever, and Geoffrey E. Hinton. 2015. Grammar as a Foreign Language. In Advances in Neural Information Processing Systems 28, pages 2773 2781 .

Taro Watanabe and Eiichiro Sumita. 2015. Transitionbased Neural Constituent Parsing. In Proceedings of the 53rd Annual Meeting of the Association for Computational Linguistics and the 7th International Joint Conference on Natural Language Processing of the Asian Federation of Natural Language Processing, pages 1169-1179.
Yang Wei, Yuanbin Wu, and Man Lan. 2020. A Spanbased Linearization for Constituent Trees. In Proceedings of the 58th Annual Meeting of the Association for Computational Linguistics, pages $3267-$ 3277.

Naiwen Xue, Fei Xia, Fu-Dong Chiou, and Martha Palmer. 2005. The Penn Chinese TreeBank: Phrase structure annotation of a large corpus. Natural Language Engineering, 11(2):207-238.

Kaiyu Yang and Jia Deng. 2020. Strongly Incremental Constituency Parsing with Graph Neural Networks. In Advances in Neural Information Processing Systems 33: Annual Conference on Neural Information Processing Systems 2020.

Zhilin Yang, Zihang Dai, Yiming Yang, Jaime G. Carbonell, Ruslan Salakhutdinov, and Quoc V. Le. 2019. XLNet: Generalized Autoregressive Pretraining for Language Understanding. CoRR, abs/1906.08237.

Yu Zhang, Houquan Zhou, and Zhenghua Li. 2020. Fast and Accurate Neural CRF Constituency Parsing. In Proceedings of the Twenty-Ninth International Joint Conference on Artificial Intelligence, pages 4046-4053.

Yang Zhao, Jiajun Zhang, Zhongjun He, Chengqing Zong, and Hua Wu. 2018. Addressing Troublesome Words in Neural Machine Translation. In Proceedings of the 2018 Conference on Empirical Methods in Natural Language Processing, pages 391-400.

Junru Zhou and Hai Zhao. 2019. Head-Driven Phrase Structure Grammar Parsing on Penn Treebank. In Proceedings of the 57th Conference of the Association for Computational Linguistics, pages 23962408.

Muhua Zhu, Yue Zhang, Wenliang Chen, Min Zhang, and Jingbo Zhu. 2013. Fast and Accurate ShiftReduce Constituent Parsing. In Proceedings of the 51st Annual Meeting of the Association for Computational Linguistics, pages 434-443.

Arnold M. Zwicky. 1985. Heads. Journal of Linguistics, 21(1):1-29. 

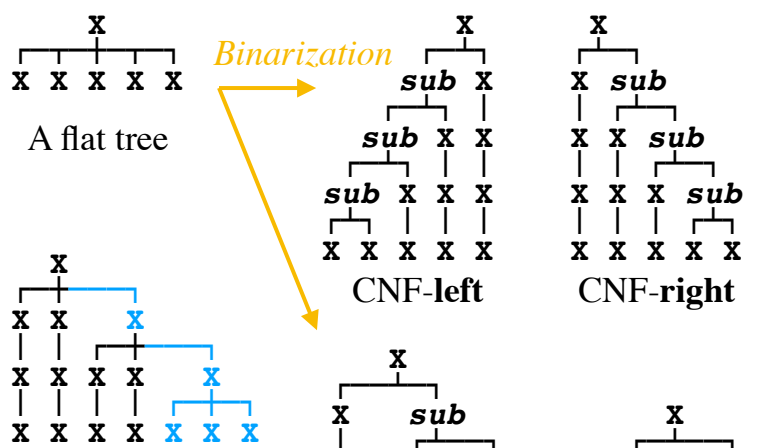

A multi-branching tree with a single dependency core: an $O\left(n^{2}\right)$ case

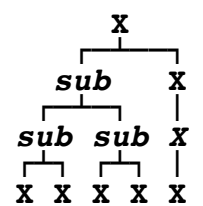

nCNF-midout

Figure 12: Examples used in the content. A flat tree is binarized with four factors. The binarization of CNFleft, $\mathrm{CNF}$-right, or $\mathrm{nCNF}$-midin creates binary trees with a single dependency core (i.e., a single $s u b$ thread), whose $O\left(n^{2}\right)$ complexity is the same with the tree without binarization in the lower left. Most nodes are relaying nodes. Meanwhile, nCNF-midout enables concurrent phrases with $O(n)$ complexity (i.e., multiple $s u b$ threads). However, the division tends to break a constituent into ungrammatical pieces, which confuses the model and does not lead to improvement.
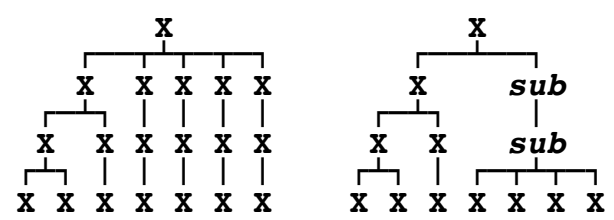

Figure 13: Adding $s u b$ nodes to make flat structure more efficient. Using the strategy as a new dynamic dataset also brings multi-branching model $\mathbf{M}$ a stable accuracy improvement with an F1 score of 92.36 on PTB. However, it has nothing to do with linguistic properties. We save it for a future study.

\section{A Appendices}

\section{A.1 Compression Ratios and Linearity}

Figure 12 presents examples for tree binarization and the worst case of $O\left(n^{2}\right)$ complexity. Figure 14 shows the overall linear data complexities in the three languages. Figures $15 \& 16$ and Table 9 indicate that, given a language and a factor, the compression ratio is stable and seldom affected by the sentence length.

The regressions for PTB and CTB show weak $O\left(n^{2}\right)$ tendencies; the quadratic coefficients can be either positive or negative. Meanwhile, KTB falls into the worst case, as shown in Figure 14. This is because KTB trees tend to have a flat structure

on the right side of parses, as illustrated in Figure 17. Relaying nodes in the flat structure never combine until the final layer, creating strong $O\left(n^{2}\right)$ tendencies. As a result, all KTB datasets fall into the worst case, especially when binarized with the CNF-left factor.

A preprocess that groups the flat structure into the $s u b$ category can prevent considerable quadratic impacts on all datasets. All $O\left(n^{2}\right)$ tendencies are largely weakened across three corpora, and all linear coefficients drop significantly, as illustrated on the right of Figure 14. The preprocess cannot eradicate the worst case in KTB. However, all linear coefficients' magnitudes are at least hundreds of times larger than those of the quadratic terms. In our sub-quadratic case, 200 words lead to approximately $1.5 \mathrm{~K}$ nodes. Meanwhile, a sentence with $n$ words has a triangular chart with $\frac{n(n+1)}{2}$ nodes, whose quadratic coefficient is 0.5 . In this case, 200 words lead to approximately $20 \mathrm{~K}$ nodes.

\section{A.2 Experiment Setting}

The treebanks PTB and CTB have been widely used for experiments. For PTB, sections 2-21 were used for training, section 22 for development, and section 23 for testing. For CTB, articles 001-270 and 440-1151 were used for training, 301-325 for development, and 271-300 for testing. There is no widely accepted data split for the KTB corpus, except for some probabilistic divisions, because KTB contains mixed data from sources such as newswires, book digests, and Wikipedia. We randomly reserved 2,075 samples for development, 1,863 samples for testing, and the remaining 3.3 million as training samples. Few sentences in the training sets were longer than 100 words (3 of $40 \mathrm{~K}$ in PTB; 96 of $17 \mathrm{~K}$ in CTB; 55 of $33 \mathrm{~K}$ in KTB). Frozen English (wiki.en.bin), Chinese (cc.zh.300.bin), and Japanese (cc.ja.300.bin) embeddings were used for PTB, CTB, and KTB, respectively ${ }^{7}$. We fed fastText with the PTB text to train cbow instead of skipgram embeddings for B/E with their default settings for 50 epochs.

The batch size was 80 , and sentences longer than 100 words were excluded for the triangular data to avoid out-of-memory (OOM) errors on a single GeForce GTX 1080 Ti with 11 GB. We froze XLNet to train our model and then tuned XLNet from the 5-th epoch. We doubled the batch size at the inference phase to 160 .

\footnotetext{
${ }^{7}$ https: / / fasttext.cc/
} 


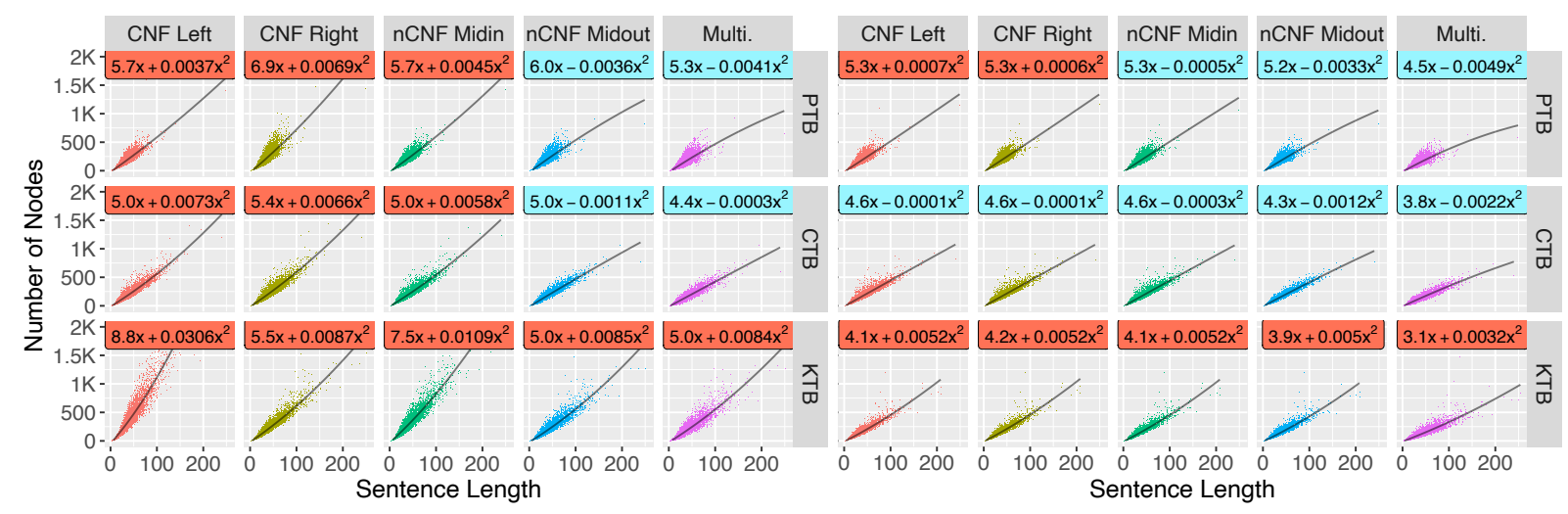

Figure 14: Left: the empirical complexities related to Figures $15 \& 16$. Linear regressions are shown on a light blue background when the quadratic terms are negative. Right: resultant complexities after a preprocessing that groups the flat structure into sub-constituent before stratification. (See Figure 13.)

\begin{tabular}{cccccc}
\hline Factor & Left & Right & Midin & Midout & Multi. \\
\hline \multicolumn{5}{c}{ All layers } \\
PTB & $0.77 \pm 0.11$ & $0.79 \pm 0.11$ & $0.78 \pm 0.11$ & $0.77 \pm 0.11$ & $0.73 \pm 0.20$ \\
CTB & $0.77 \pm 0.11$ & $0.77 \pm 0.11$ & $0.76 \pm 0.11$ & $0.74 \pm 0.11$ & $0.70 \pm 0.20$ \\
KTB & $0.82 \pm 0.12$ & $0.75 \pm 0.12$ & $0.79 \pm 0.12$ & $0.73 \pm 0.12$ & $0.69 \pm 0.29$ \\
\hline \multicolumn{5}{c}{ Layers longer than 40} \\
PTB & $0.78 \pm 0.04$ & $0.80 \pm 0.04$ & $0.77 \pm 0.04$ & $0.74 \pm 0.04$ & $0.69 \pm 0.08$ \\
CTB & $0.79 \pm 0.04$ & $0.80 \pm 0.04$ & $0.78 \pm 0.04$ & $0.77 \pm 0.04$ & $0.76 \pm 0.07$ \\
KTB & $0.90 \pm 0.04$ & $0.80 \pm 0.05$ & $0.86 \pm 0.04$ & $0.77 \pm 0.06$ & $0.84 \pm 0.07$ \\
\hline
\end{tabular}

Table 9: Mean and standard deviation of compression ratios of Figures $15 \& 16$. Longer layers have converged deviations. The last column came from the multi-branching treebanks without a binarizing factor.

We used the Adam optimizer with a default learning rate of $10^{-3}$, while we opted for the XLNet's Adam hyperparameters when tuning the pre-trained XLNet (e.g., their learning rate was $10^{-5}$ ). We adopted a warm-up period for one epoch and a linear decrease after the 15-th decrease since the last best evaluation. The recurrent dropout rate was 0.2 ; other dropout probabilities for FFNNs were set to 0.4 . For model selection, the training process terminated when the development set did not improve above the highest score after 100 consecutive evaluations. The Evalb program ${ }^{8}$ was used for F1 scoring.

We demonstrated score profiles for our main models in Table 10. The discrepancy in F1 scores and difference between precision and recall are relatively small on the PTB development and test sets.

\section{A.3 Variants of Binary Compose}

If we choose the relay instruction in line 12 of Algorithm 2, additive vector compositionality is retained (Mikolov et al., 2013) as the naïve ADD

\footnotetext{
${ }^{8}$ https://nlp.cs.nyu.edu/evalb/
}

\begin{tabular}{lccrcc}
\hline Input & \multicolumn{3}{c}{ Development } & \multicolumn{2}{c}{ Test } \\
Comp. & M. & F1 & $P-R$ & F1 & $P-R$ \\
\hline Frozen & B & 92.50 & 0.00 & 92.54 & +0.56 \\
fastText & M & 92.10 & -0.35 & 92.10 & -0.03 \\
\hline Tuned & B & 95.64 & -0.05 & 92.72 & +0.19 \\
XLNet & M & 95.34 & -0.15 & 92.44 & +0.30 \\
\hline
\end{tabular}

Table 10: F1 scores and differences in precision and recall $(P-R)$ on the PTB development and test sets.

variant in lines 5-6 of Algorithm 4. The model can infer a full tensor tree; however, ADD causes the vector magnitude to increase with the tree height cumulatively. This is unwanted in the recurrent or recursive neural network.

Therefore, we examined a learnable $\mathrm{FFNN}_{\text {multi }}$ with Sigmoid activation to perform gate-style interpolation in five variants $\mathbf{N S}, \mathbf{N V}, \mathbf{C S}, \mathbf{C V}$, and BV as described in lines 8-17. When a variant takes no input and produces a scalar interpolation parameter $\lambda$, we consider this case NS. (" $\varnothing$ " is a placeholder for no input.) Meanwhile, CV indicates concatenated input and vectorized interpolation. $\mathbf{B} \mathbf{V}$ is a variant that involves a biaffine tensor 

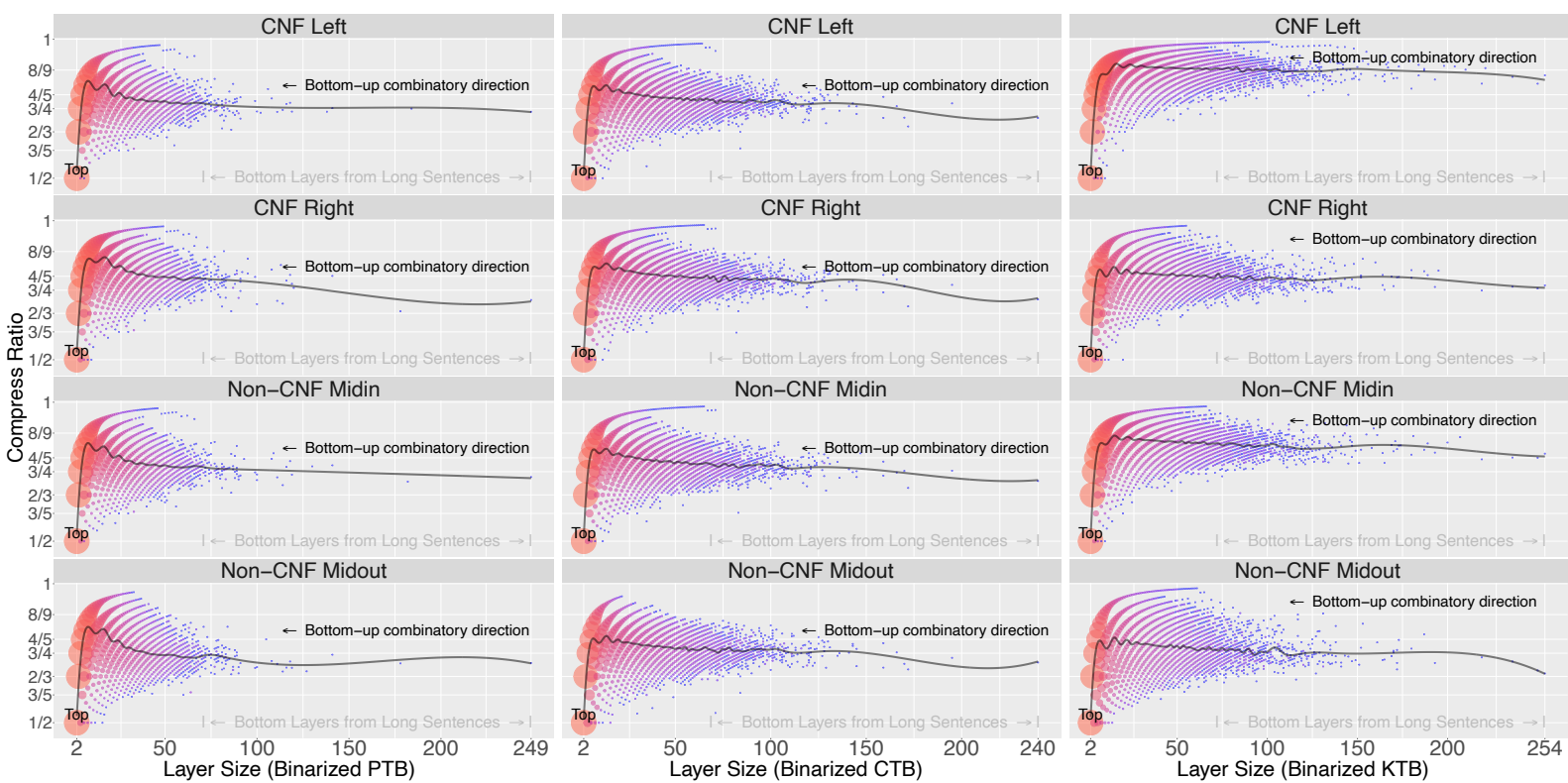

Figure 15: Binarized corpora with four factors. Curved tiers can be observed in each plot. For example, the leftmost tier is composed of $\frac{n-1}{n}$ (followed by $\frac{n-2}{n}, \frac{n-3}{n}$, and so on). The dots in this tier range from a high compression ratio of 0.5 to the least efficient ones in their corpus. Efficient dots are more populated, judging by their sizes and colors. All statistics yield stable means, which are also presented in Table 9.
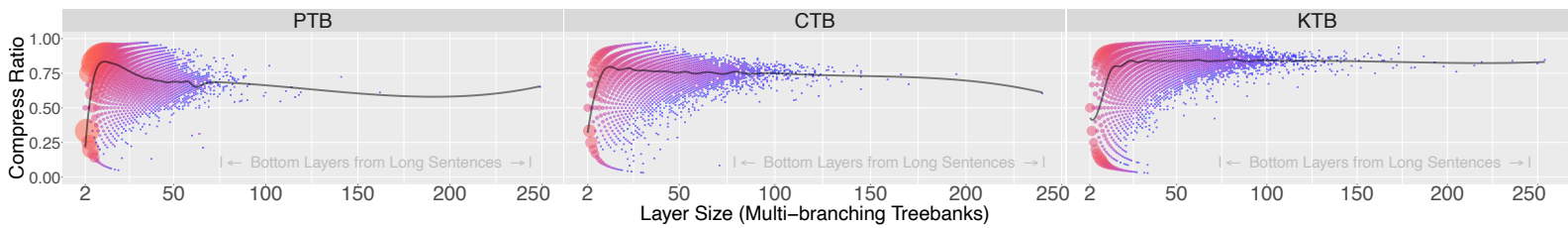

Figure 16: Multi-branching corpora. Curved tiers appear more symmetric and all statistics still yield stable means.

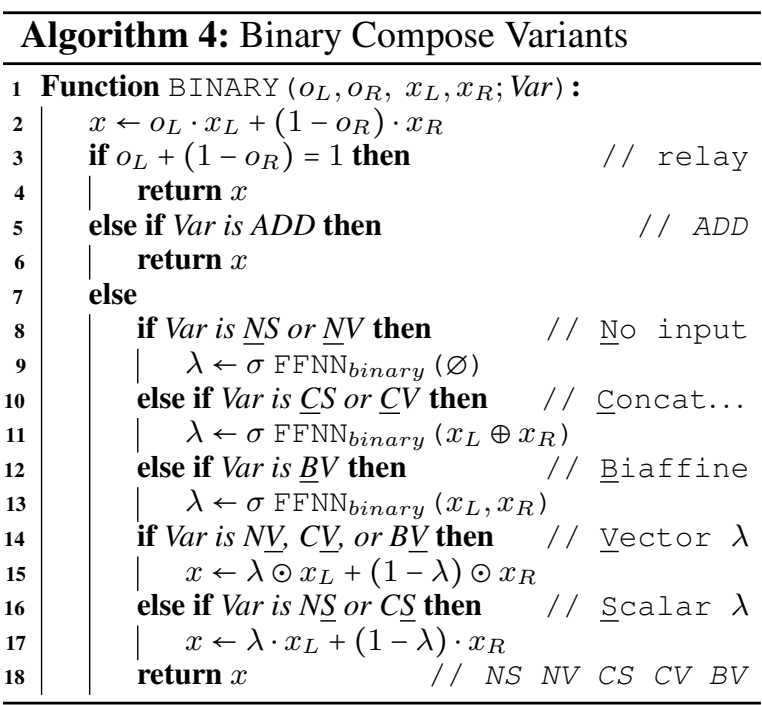

operation. $\mathbf{C V}$ is our default BINARY variant; the experiments for these variants are presented in Table 11.

In terms of the F1 score, the most competitive variants of $\mathbf{C V}$ are $\mathbf{B V}$ and $\mathbf{N V}$, suggesting that fine

\begin{tabular}{ccc}
\hline Var & Specification & F1 \\
\hline BV & Biaffine inputs for vector $\lambda$. & $\underline{92.53}$ \\
CV & $x_{L} \oplus x_{R}$ as input for vector $\lambda$. & $\mathbf{9 2 . 5 4}$ \\
CS & $x_{L} \oplus x_{R}$ as input for scalar $\lambda$. & 91.83 \\
NV & No input; bias vector $\lambda$. & 92.36 \\
NS & No input; bias scalar $\lambda$. & 91.95 \\
ADD & $x_{L}+x_{R}$ & 91.86
\end{tabular}

Table 11: Compositionality of the BINARY function.

interpolation can effectively facilitate vector compositionality. The similarity in results of CS, NS, and ADD validate this suggestion. This indicates that vector compositionality is not as trivial as an additive function at the scalar level, and a matrix operation is sufficient. BV is the costliest variant with a tensor operation that runs very slowly (30 sents/sec).

\section{A.4 Recovering Symbolic Tree}

To obtain the final tree representation, we initialized the working place with leaves of words and 

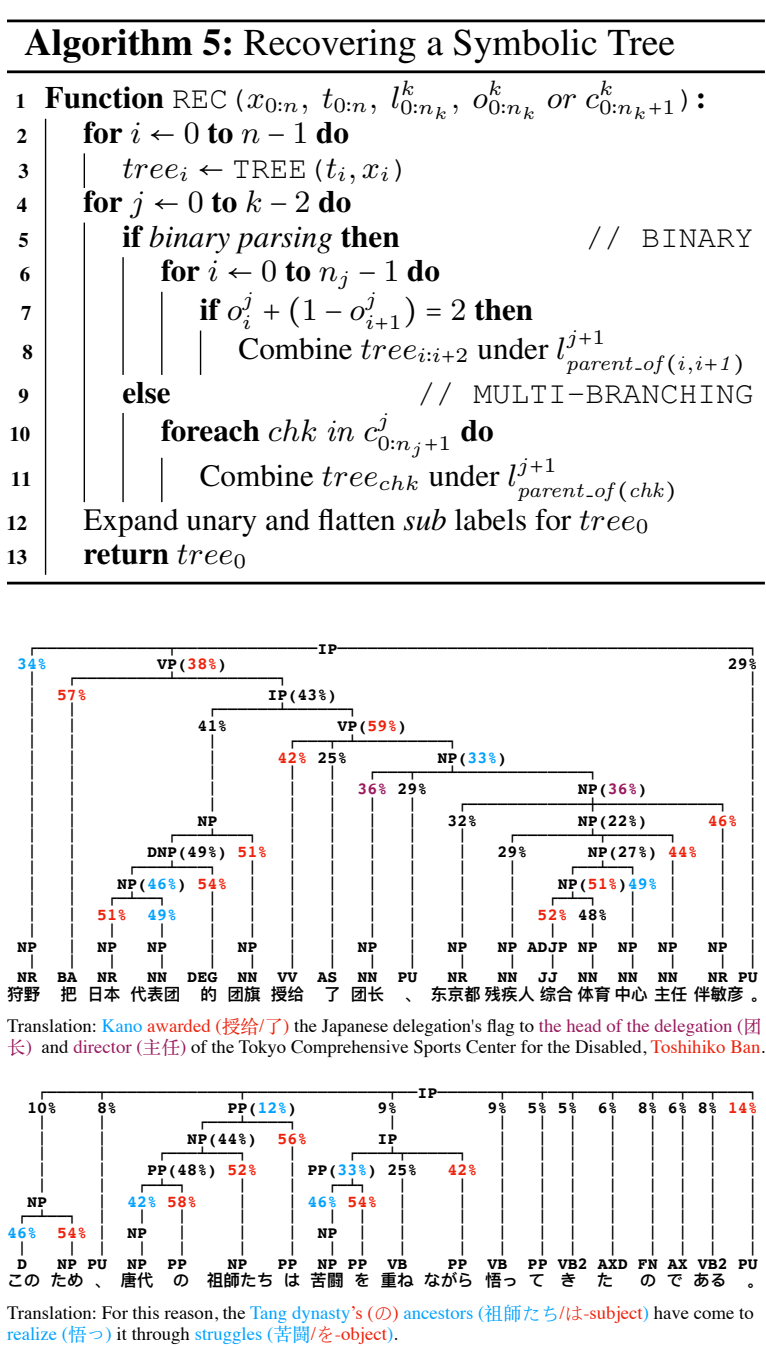

Figure 17: Chinese (top) and Japanese (bottom) parses from the multi-branching model.

predicted POS tags. Two symbolic rules were used to modify the labels and construct sub-trees, as described in Algorithm 5. 1) The collapsed unary branches were expanded to their original structure by splitting at the plus marks (e.g., SBAR+S into SBAR and S). 2) 'the label is a sub' excluded the repeated labels and relayed sub-trees. These rules enabled a single tree $_{0}$ as the final output.

\section{A.5 Chinese and Japanese Headedness}

Figure 17 presents two non-English parses from the multi-branching model. Both the Chinese and Japanese languages possess functional markers that receive high attention (percentage and words in red), such as the second character tagged with $B A$ in Chinese, and Japanese case markers tagged with PP. Interestingly, the Chinese verb (i.e., the one meaning "awarded") received the highest attention, whereas Japanese verbs (i.e., two sub-words tagged with VB) did not. We supposed the reason behind this is that Japanese sentences drop the VBs and other heads more often than Chinese. The coordinated NPs in the Chinese parse (i.e., two words meaning "head" and "director") received equal attention weights.

Moreover, two trees show their branching tendencies: Chinese is midin-alike; Japanese is a leftbranching language, and KTB has a large flat structure on the right. 